\title{
Clinical evaluation of Dinamap 845 automated blood pressure recorder
}

\author{
J H SILAS, A T BARKER, * L E RAMSAY \\ From the Departments of Therapeutics and Medical Physics ${ }^{\star}$, Hallamshire Hospital, Sheffield
}

SUMMARY The Dinamap 845 blood pressure recorder has been evaluated over a wide range of blood pressure by comparison with the Hawksley random zero sphygmomanometer in 32 subjects, six of whom had a cardiac arrhythmia. Group mean readings for systolic and phase 5 diastolic pressure were almost identical but Dinamap diastolic values were on average significantly lower (mean difference $3.4 \mathrm{mmHg}$ ) than phase 4 diastolic readings obtained with the Hawksley machine. Correlations between readings with the two instruments were high but the slopes and intercepts of the regression for systolic but not diastolic pressure were significantly different from unity and zero, respectively. The Dinamap is easy to use, portable, and capable of rejecting some motion artefact. Its major disadvantage is that the systolic blood pressure measurement is limited to a maximum of $210 \mathrm{mmHg}$, a point not made clear in the manufacturer's literature. Nevertheless, the Dinamap 845 is acceptable for blood pressure determinations in subjects who are normotensive or who have mild hypertension.

The mercury sphygmomanometer remains the standard device for measuring blood pressure in clinical practice and in clinical or epidemiological research. Modified versions such as the Hawksley random zero and London School of Hygiene sphygmomanometers have reduced the problem of observer bias in measurement, but inaccuracy resulting from differences between observers remains. To eliminate this many semiautomated blood pressure recording devices have been introduced. Their use has been criticised on the grounds that they are inaccurate, or that their accuracy has been poorly documented. ${ }^{1}$ We have evaluated the Dinamap 845 automated blood pressure recorder, using the Hawksley random zero sphygmomanometer as the standard for comparison.

\section{Methods}

\section{RECORDING DEVICES}

The Dinamap 845 (Fig. 1) is designed to monitor systolic, diastolic, and mean arterial pressure, and pulse rate. Manual determinations can be made using a switch on the front panel, or repeated fully automatic measurements can be obtained at chosen intervals between one and eight and a half minutes (since changed by the manufacturers to one to 16 minutes). The machine operates on an oscillometric Received for publication 15 August 1979 principle measuring pressure fluctuation within a cuff, ${ }^{2}$ and has no microphone or external transducer. Cuffs of three sizes are provided, enabling measurements to be made in obese patients or on the lower limbs. When the device is switched on the cuff is inflated automatically to $160 \mathrm{mmHg}$, and with subsequent automatic readings the cuff is pumped to a pressure approximately $34 \mathrm{mmHg}$ higher than the previously determined systolic pressure. The cuff deflates in steps of 3 to $6 \mathrm{mmHg}$ and the cuff pressure at each step is momentarily displayed. Errors from limited and intermittent motion artefact are detected and excluded using pulse amplitude averaging and error rejection programmes. When artefacts are detected the incremental cuff deflation cycle is halted until the artefact ceases, or until 60 seconds have passed when an alarm sounds and the cuff deflates completely. Recordings are interpolated between pressure steps during cuff deflation, and the step size does not apparently limit the accuracy of the measurements. Readings are displayed digitally on the front panel, and may also be recorded graphically using the Dinamap 950, which we did not test. The alarm mentioned above also sounds when mean arterial pressure is outside preselected high and low limits, and in the event of abnormal operating conditions, for example internal machine faults or measurements taking longer than three minutes. 


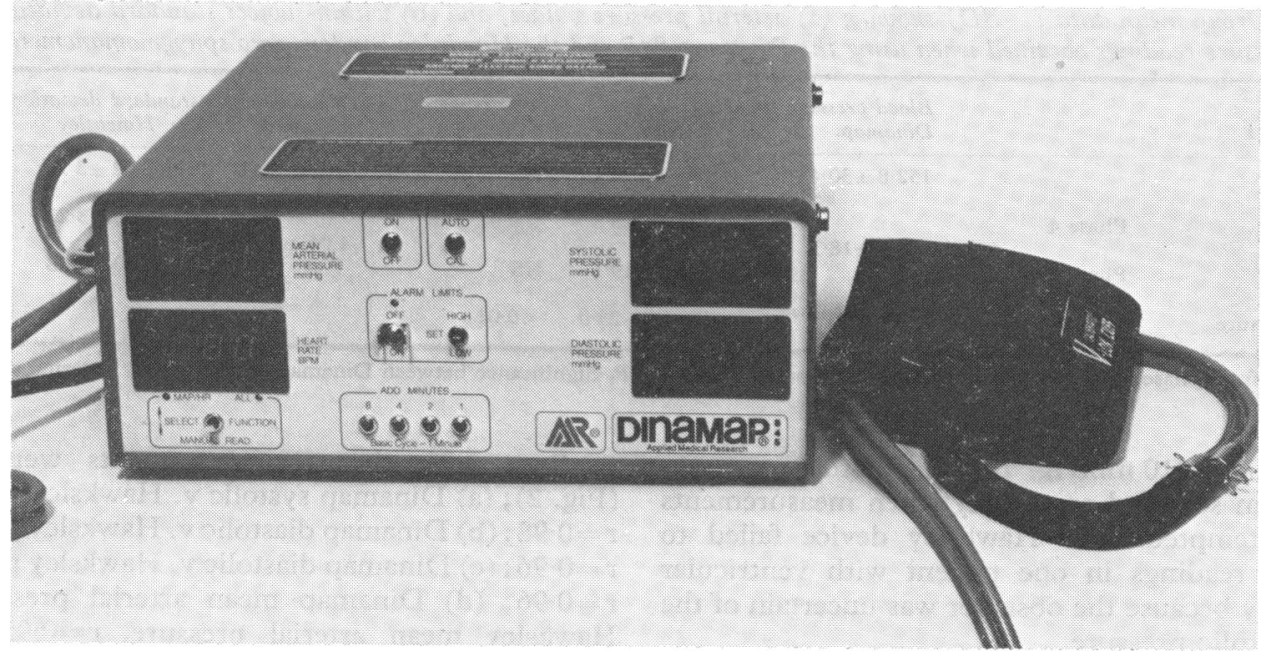

Fig. 1 The Dinamap 845 blood pressure recorder.

The Dinamap 845 weighs $7: 5 \mathrm{~kg}$, is portable, and is powered by mains electricity. A device using the same principles, but recording only mean arterial pressure and heart rate, has been tested against direct intra-arterial measurements. ${ }^{3}$

The Hawksley random zero sphygmomanoneter is in essence a standard mercury sphygmomanometer with a zero muddle device to reduce observer bias. ${ }^{4}$ The deflation rate was regulated to 2 to $3 \mathrm{~mm} / \mathrm{s}$. Measurements with this machine correlate well with intra-arterial pressures. ${ }^{5}$

\section{Subjects}

Twenty-seven patients and five healthy subjects were selected to provide a wide spread of blood pressure measurements. Nine of the patients had some abnormality of cardiac rhythm (atrial fibrillation in six, ventricular extrasystoles in three). One patient had an auscultatory gap, and two patients had persistence of Korotkoff sounds to $0 \mathrm{mmHg}$. In the latter patients phase 5 diastolic measurements were taken to be identical to phase 4 readings for the purpose of analysis. The range of arm girths was 18 to $34 \mathrm{~cm}$, and a standard cuff size $(23.5 \times$ $13.5 \mathrm{~cm}$ ) was used in all patients with both machines.

\section{PROCEDURE}

After five minutes recumbent four pairs of simultaneous measurements were taken, with the Dinamap on one arm, the Hawksley on the other. The machines were changed to the other arm after each measurement. The Dinamap was used on the right arm first in alternate subjects. Pressure measurements were read to the nearest $1 \mathrm{mmHg}$ with both devices. Systolic, diastolic phase 4, and diastolic phase 5 pressures were recorded with the Hawksley, and mean arterial pressure was calculated as diastolic phase 5 plus one-third pulse pressure. Systolic, diastolic, and mean arterial pressures were read from the Dinamap. The observers did not communicate with each other until all recordings in one subject were complete. The mean of the four readings in each subject was calculated for each machine, so that the comparison of the machines was not biased by variations in blood pressure with time or between the two arms. The mean results for each recorder were compared by Student's $t$ test for paired observations, and product-moment correlation coefficients were calculated. The calculated regression lines were examined to determine whether their slopes deviated significantly from unity, and their intercepts from zero.

\section{Results}

\section{FAILURE TO OBTAIN RECORDINGS}

Satisfactory measurements could not be obtained in three subjects, with failure of the Dinamap 845 in two, and failure of the Hawksley (or, more precisely, of the auscultatory method) in one. The Dinamap was consistently incapable of measuring the blood pressure in one patient with atrial fibrillation, though it recorded satisfactorily in the other five patients with this arrhythmia. It also failed to record in one patient with a systolic blood pressure of $260 \mathrm{mmHg}$, and we discovered subsequently that the device was incapable of measuring pressures. 
Table Group mean data ( $\pm S D$ ) showing (a) arterial pressure values, and (b) within-subject standard deviation in blood pressure readings obtained when using the Dinamap 845 and the Hawksley random zero sphygmomanometer

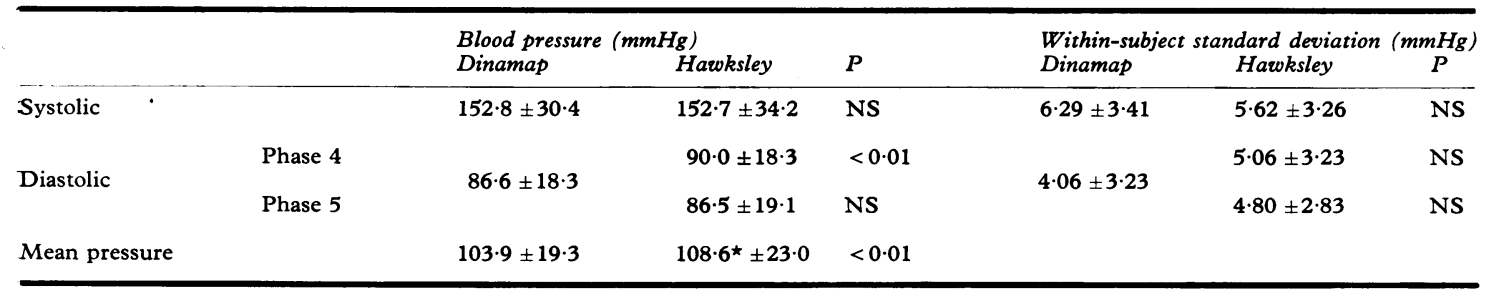

$\star$ Derived from phase 5 diastolic blood pressure $+\frac{1}{3}$ pulse pressure. P, Significance between Dinamap and Hawksley determinations.

higher than $210 \mathrm{mmHg}$. In both of these patients the alarm sounded repeatedly when measurements were attempted. The Hawksley device failed to provide readings in one patient with ventricular bigeminy because the observer was uncertain of the true systolic pressure.

\section{ACCURACY OF DINAMAP 845}

The data for the remaining 29 subjects are shown in the Table. The Dinamap systolic and diastolic pressures were virtually identical to the Hawksley systolic and phase 5 diastolic pressures, respectively. Dinamap diastolic pressures were on average 3.4 $\mathrm{mmHg}$ lower than Hawksley phase 4 readings $(P<0.001)$. The mean arterial pressure read from the Dinamap was $4.7 \mathrm{mmHg}$ lower than mean arterial pressure calculated from the Hawksley measurements $(P<0.002)$. Correlations between

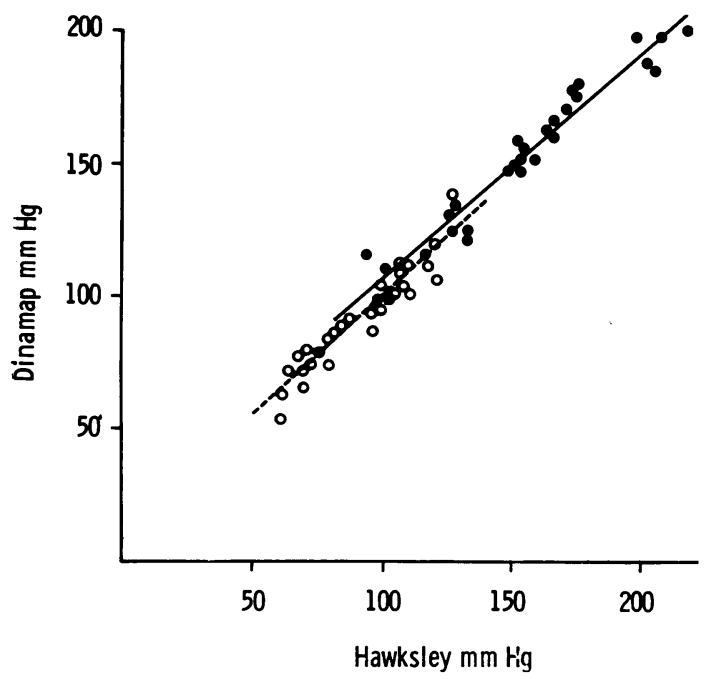

Fig. 2 Correlation between blood pressure values obtained using the Hawksley sphygmomanometer and the Dinamap 845. Closed circles, systolic $r=0.98$, slope 0.88, intercept $19 \mathrm{mmHg}$; open circles, diastolic (phase 5) $r=0.96$, slope 0.91, intercept $6 \mathrm{mmHg}$. readings with the two instruments were high (Fig. 2); (a) Dinamap systolic v. Hawksley systolic, $\mathbf{r}=0.98$; (b) Dinamap diastolic v. Hawksley phase 5, $r=0.96$; (c) Dinamap diastolic v. Hawksley phase 4, $r=0.96$; (d) Dinamap mean arterial pressure v. Hawksley mean arterial pressure, $r=0.95$. The slopes and intercepts were (a) $0.88,19 \mathrm{mmHg}$; (b) $0.91,6 \mathrm{mmHg}$; (c) $0.91,8 \mathrm{mmHg}$; (d) 0.79 , $18 \mathrm{mmHg}$, respectively. Significant differences from unit slope and zero intercept $(\mathbf{P}<0.01)$ were noted only for systolic (a) and mean (d) blood pressure. In an individual, readings obtained by the two instruments were equally consistent.

PERFORMANCE OF DINAMAP 845

Measurements with the Dinamap were usually complete in about 40 seconds, on average slightly quicker than the Hawksley readings. Placement of the cuff did not appear to be critical. The alarm system seemed to function well. The performance of the machine we tested deviated in two important respects from the specifications given in the manufacturer's literature, and described in the Methods section. Most surprising was the failure of the device to measure pressures above 210 $\mathrm{mmHg}$. This is not mentioned in the literature, though the manufacturers were in fact aware of it. The cuff deflation steps in the instrument were 8 to $10 \mathrm{mmHg}$, and not the 3 to $6 \mathrm{mmHg}$ quoted.

\section{Discussion}

The Dinamap 845 faithfully reflected systolic and diastolic blood pressure readings within the range 50 to $200 \mathrm{mmHg}$. Though the regression line for systolic blood pressure deviated from a unit slope with zero intercept, the difference did not seem clinically important in the range of blood pressure tested (Fig. 2). The close agreement with phase 5 as opposed to phase 4 diastolic values is no disadvantage since this is the criterion used in most epidemiological studies. ${ }^{67}$ The mean arterial pressure did not correspond closely but it is known 
that the derived values obtained by the mercury manometer are an approximation ${ }^{8}$. Mean arterial pressure obtained with an earlier Dinamap model corresponded closely with intra-arterial pressures. ${ }^{3}$

In general, blood pressure measurements were not hampered by arrhythmias, a silent interval, or persistence of Korotkoff sounds to zero. There were no grossly erroneous readings and the alarm system functioned well.

The machine is light and therefore easily portable, quick, and simple to use and required no ultrasonic gel. As cuff position is not critical it should be accurate in the hands of an inexperienced observer, and we made no effort to effect exact cuff placement.

The major disadvantage of the device is that systolic blood pressure measurement is limited to a maximum of $210 \mathrm{mmHg}$. This limits the machine's use to subjects who do not have severe hypertension. Furthermore, it is necessary to exclude the first reading in subjects with a systolic blood pressure above $155 \mathrm{mmHg}$. This is not a significant defect when repeated blood pressure measurements are required but is a disadvantage with single determinations. In such a situation, however, a potential error may be accurately predicted from the display of cuff pressure during each deflation step.

The method used to eliminate errors arising from motion artefact leads to a delay in blood pressure determination. This is likely to be a problem when there is continuous movement artefact with brief pauses that enable the cuff to be deflated slightly. In such an event it is possible for the cuff to be kept above the subject's systolic pressure for up to 3 minutes. Decreasing the constant pressure alarm criterion from 60 seconds would make this unusual problem less likely to cause discomfort.

The Dinamap 845 has a useful place for repeated blood pressure determinations in subjects with normal blood pressure or with mild hypertension. It may prove useful in an intensive care unit though the machine has yet to be tested at blood pressure values below $90 / 50 \mathrm{mmHg}$. The consistency between different machines and of an individual instrument over a period of time also remains to be demonstrated. Finally, deficiencies in the manufacturer's literature need to be rectified.

\section{References}

${ }^{1}$ Raftery EB. The methodology of blood pressure recording. Br f Clin Pharmacol 1978; 6: 193-202.

${ }^{2}$ Richardson OW, Honour AJ, Fenton GW, Scott FH, Pickering GW. Variations in arterial pressure throughout the day and night. Clin Sci 1964; 26: 445-60.

${ }^{3}$ Ramsey M III. Non-invasive automatic determination of mean arterial pressure. Med Biol Eng Comput 1979; 17: $11-8$.

${ }^{4}$ Wright BM, Dore CF. A random zero sphygmomanometer. Lancet 1970; 1: 337-8.

${ }^{5}$ Hunyor SN, Flynn JM, Cochineas C. Comparison of performance of various sphygmomanometers with intra-arterial blood pressure readings. $\mathrm{Br}$ Med $\mathcal{F} 1978$; 2: 159-62.

${ }^{6}$ Veterans Administration Co-operative Study Group on Antihypertensive Agents. Effects of treatment on morbidity in hypertension. $\mathcal{F} A M A 1967$; 202: 1028-34.

${ }^{7}$ Medical Research Council Working Party on Mild to Moderate Hypertension. Randomised controlled trial of treatment for mild to moderate hypertension: design and pilot trial. $\mathrm{Br} \mathrm{Med} \mathcal{F} 1977 ; 1: 1437-40$.

${ }^{8}$ Thulin T, Andersson G, Schersten B. Measurement of blood pressure-a routine test in need of standardization. Postgrad Med f 1975; 51 : 390-5.

Requests for reprints to Dr J H Silas, Department of Therapeutics, Hallamshire Hospital, Glossop Road, Sheffield S10 2JF. 